Goldschmidt 2021 Abstract

https://doi.org/10.7185/gold2021.8139 differences in microbial diversity between Solfatara and Pisciarelli.

\section{Subsurface Fluid Geochemistry Shape Microbial Community Structure and Function at Solfatara-Pisciarelli Hydrothermal Systems, Italy.}

\author{
IFEOMA R UGWUANYI ${ }^{1}$, MIHAELA GLAMOCLIJA ${ }^{1}$, \\ ANDREW STEELE ${ }^{2}$, MARILYN FOGEL ${ }^{3}$, ROXANE \\ BOWDEN $^{4}$, GIUSEPPE DE NATALE ${ }^{5}$, CLAUDIA TROISE ${ }^{6}$, \\ RENATO SOMMA ${ }^{5}$, MONICA PIOCHI ${ }^{6}$ AND ANGELA \\ MORMONE $^{6}$
}

${ }^{1}$ Rutgers University-Newark

${ }^{2}$ Carnegie Institution for Science

${ }^{3}$ University of California Riverside

${ }^{4}$ Carnegie Institution of Washington (former)

${ }^{5}$ Istituto Nazionale di Geofisica e Vulcanologia (INGV)

${ }^{6}$ Istituto Nazionale di Geofisica e Volcanologia

Presenting Author: iru1@scarletmail.rutgers.edu

Geochemical differences in hydrothermal systems are strongly influenced by subsurface fluid circulations and mixing, and processes such as subsurface boiling, phase separation, and water-rock interaction influence the availability of electron donors/acceptors capable of supporting microbial metabolism in the hydrothermal systems. In this study, we investigate how geochemical variations that arise from subsurface fluid circulations drive the ecology of two adjacent hydrothermal systems. Solfatara Crater (Naples, Italy) contains active hydrothermal system, and on the outside wall of the crater is another active hydrothermal site, Pisciarelli. The geochemical differences in fluids among these systems is derived from subsurface water-rock interaction. Solfatara hydrothermal fluids produce an acidic and oxidizing ( $\mathrm{pH} 1.0$, Eh 198 to 330) environment while Pisciarelli is acidic and reducing $(\mathrm{pH} 1.5$ to 3.0, Eh -628 to -485 ). Pisciarelli and Solfatara had average $\delta^{34} \mathrm{~S}$, $\delta^{13} \mathrm{C}$, and $\delta^{15} \mathrm{~N}$ values that was indicative of a magmatic source for carbon, sulfur, and nitrogen. Overall, Pisciarelli contains three times more $\mathrm{NH}_{4}^{+}$and $\mathrm{SO}_{4}{ }^{2-}$ than Solfatara. Principal Component Analysis (PCA) showed that Solfatara differed from Pisciarelli by $\mathrm{Eh}, \mathrm{NO}_{3}{ }^{-}$and $\mathrm{PO}_{4}{ }^{3-}$. Metagenomic sequencing revealed that archaea were dominant in water and mud samples from Pisciarelli, while bacteria were dominant in soil samples from Pisciarelli and all the Solfatara samples. Functional genes showed differential abundance in Pisciarelli water and mud samples compared to all Solfatara samples and soil samples from Pisciarelli. Genes such as aprB and nrf involved in sulfur oxidation and nitrite reduction, respectively were abundant in Pisciarelli mud and water samples compared to all Solfatara samples and soil samples from Pisciarelli. Functional genes that were abundant in Solfatara samples and soil sample from Pisciarelli include norB and fhs involved in denitrification and acetate oxidation, respectively. Cluster analysis of functional genes from the three domains of life support this distinction of Pisciarelli water and mud communities from Pisciarelli soil and all the Solfatara samples. Overall, the geochemical difference we observed in hydrothermal fluids are likely responsible for the 